\title{
Réflexions sur trois épisodes de la vie de Louis Pasteur vus au travers du film de William Dieterle (1936)
}

\section{Reflections on Three Episodes of Louis Pasteur's Life as Seen in the William Dieterle's Movie (1936)}

\author{
C.T. Daniel-Ribeiro $\cdot$ M.M. Lima · J.-F. Pays \\ Reçu le 29 décembre 2018; accepté le 4 avril 2019 \\ (C) Société de pathologie exotique et Lavoisier SAS 2019
}

\begin{abstract}
Résumé Malgré les critiques et les réserves dont il est l'objet aujourd'hui, Louis Pasteur peut être considéré comme l'un des scientifiques les plus importants de ces deux derniers siècles en matière de santé publique, même si les apports des nombreux hommes de science qui l'ont précédé ont largement contribué aux succès qu'il obtint sans trop se soucier des règles de déontologie et d'éthique actuellement en vigueur dans le monde de la recherche et de la médecine. Il a mis définitivement à bas, par ses expériences, la « théorie de la génération spontanée » en vigueur depuis l'Antiquité, validé celle « des germes ou microbes », édicté les premières règles de l'asepsie tout en inspirant celles de l'antisepsie appliquée par Joseph Lister et mit au point un certain nombre de vaccinations en médecine vétérinaire puis humaine, notamment celle contre la rage, ce qui le rendit célèbre dans le monde entier. Tout cela ne s'est pas fait sans difficulté, et Pasteur s'est heurté pendant une grande partie de sa vie à l'incompréhension de ses contemporains et à l'hostilité du monde médical auquel il n'appartenait pas. Les auteurs commentent dans ce texte le film L'Histoire de Louis Pasteur de William Dieterle, tourné en 1936, en s'appuyant sur les connaissances acquises depuis cette date et en faisant la part du réel et de l'imaginé.
\end{abstract}

\footnotetext{
C.T. Daniel-Ribeiro ( $\square)$

Laboratório de Pesquisa em Malária (IOC, Fiocruz) and Centro de Pesquisa, Diagnóstico e Treinamento em Malária (Fiocruz), Rio de Janeiro, Brazil

e-mail : malaria@fiocruz.br

M.M. Lima

Laboratório de Ecoepidemiologia da Doença

de Chagas (IOC, Fiocruz), Rio de Janeiro, Brazil

J.-F. Pays

Société de pathologie exotique, hôpital Pitié-Salpêtrière,

47-83, boulevard de l'Hôpital, F-75651 Paris cedex 13, France
}

\begin{abstract}
Mots clés Louis Pasteur · Joseph Lister · Girolamo Fracastoro · Marcus von Plenciz · Wendel Holmes · Ignác Fülöp Semmelweis · Franz von Soxlhet · Joseph Meister · Asepsie $\cdot$ Antisepsie $\cdot$ Henry Toussaint $\cdot$ Anthrax $\cdot$ Rage Victor Galtier · Vaccins ' Charbon · Hétérogénie · Histoire · Histoire des sciences · William Dieterle · Biopics
\end{abstract}

\begin{abstract}
Despite the criticism and reservations made about him still nowadays, Louis Pasteur may be considered one of the most important scientists of the last two centuries in public health, even if the work of the numerous scientists who preceded him have largely contributed to the successes he obtained without following too much to the rules of deontology and ethics currently in force in the world of research and medicine. He has definitively put down, by his experiments, the "theory of spontaneous generation" in force since antiquity, validated that of "germs or microbes", enacted the first rules of asepsis, while inspiring those of the antisepsis applied by Joseph Lister, and developed a certain number of vaccinations in veterinary and human medicine, including the anti-rabies, the one which made him famous all over the world. All this was not done without difficulty and Pasteur encountered for a large part of his life the misunderstanding of his contemporaries and the hostility of the medical world to which he did not belong. The authors comment in this text the movie The Story of Louis Pasteur by William Dieterle, filmed in 1936, based on the knowledge acquired since that date and doing the part of the real and the fiction.
\end{abstract}

Keywords Louis Pasteur · Joseph Lister · Girolamo Fracastoro · Marcus von Plenciz · Wendel Holmes · Ignác Fülöp Semmelweis · Franz von Soxlhet · Joseph Meister · Henry Toussaint · Victor Galtier · Anthrax · Heterogenesis · Rabies · Vaccines · Paradigm · Asepsis · Antisepsis · History $\cdot$ History of science $\cdot$ William Dieterle $\cdot$ Biopics 
«Permettez-moi de vous révéler le secret qui m'a conduit à atteindre mon but.

Ma force repose uniquement sur ma ténacité »

Louis Pasteur

« Ne jamais être découragé pour ne pas démériter »

Oswaldo Cruz

Malgré l'existence de plusieurs documentaires sur le sujet, le plus classique des films relatant la vie de Louis Pasteur est L'Histoire de Louis Pasteur, tourné en 1936 par William Dieterle, et produit par la Warner Bros, avec Paul Muni dans le rôle-titre [5]. Au travers des connaissances accumulées pendant presque un siècle sur la vie de ce savant et en nous appuyant sur des documents qui étaient inaccessibles à l'époque du tournage, nous nous proposons d'identifier, en les commentant, les principales entorses faites dans ce film à l'Histoire dont la biographie de Pasteur fait désormais partie, tout comme le film de Dieterle, triplement oscarisé, fait partie de celle du cinéma, ne serait-ce que pour avoir lancé la mode des biopics ou films biographiques.

Le film commence à Paris en 1860, lorsque Pasteur a 38 ans. Le point central du récit expose les difficultés et l'hostilité auxquelles le scientifique dut faire face, venant à la fois du milieu médical, de l'Académie nationale de médecine et même de l'empereur Napoléon III, en raison de l'adhésion de Pasteur à la théorie des germes ou microbes dont il assurait alors la promotion, avant de l'élargir et de la refonder en y apportant les preuves scientifiques qui lui avaient manqué jusque-là. Ces difficultés et cette hostilité furent renforcées par le fait qu'il était chimiste et non médecin. Le film s'inspire certes de la vie d'un personnage historique, mais nombre des scènes qu'il nous présente ont bénéficié, en bien ou en mal, de la créativité des scénaristes et caricaturent souvent ce qui s'est réellement passé quand elles ne vont pas à son encontre.

\section{Origine des maladies et « théorie des germes »}

Un aspect fondamental à considérer, lorsqu'on analyse les origines de la " théorie des germes » et qu'on en retrace son histoire, est de disposer d'une bonne connaissance des concepts générés par les savants qui ont précédé la pensée de Pasteur ou qui ont fait progresser, parallèlement à ces concepts, les connaissances de la communauté scientifique dans le domaine de la médecine et de la biologie ou dans tous autres domaines, notamment dans ceux de la physique, de la chimie, des sciences naturelles et des technologies. Il est intéressant de noter à ce sujet le nombre de lauréats de prix Nobel qui reconnaissent avoir emprunté dans leur recherche des idées venant de sciences différentes et même parfois très éloignées de la leur. Rappelons à ce sujet que Pasteur était chimiste de formation et que ses premiers tra- vaux portèrent sur la dissymétrie moléculaire de l'acide tartrique... avant qu'il ne s'intéresse aux levures et fermentations parce qu'il pensait que celles-ci jouaient un rôle dans la dissymétrie moléculaire de l'alcool amylique.

Depuis l'aube de l'humanité, la recherche de moyens pour prévenir les maladies a été une des principales préoccupations de nombreux chercheurs. Hippocrate soulignait déjà l'importance pour un médecin de faire bouillir l'eau servant à laver les plaies et la nécessité de se laver les mains, de se couper les ongles, de les nettoyer et d'utiliser des pansements imprégnés de substances médicamenteuses pour couvrir les plaies. Mais, 400 ans après J.-C., une chape d'obscurantisme s'abattit sur l'Europe, et ce sont les faux monnayeurs que l'on se mit à faire bouillir à la place de l'eau, en même temps que l'on brûlait les sorcières! Cette chape de plomb ne s'allégea que très progressivement, et il fallut du courage pour braver les interdits, rejeter les explications simplistes de l'église et du clergé et continuer à rechercher les moyens de prévenir les maladies infectieuses, faute de pouvoir les expliquer et les guérir. $\mathrm{Au} \mathrm{XvI}{ }^{\mathrm{e}}$ siècle (1546), en pleine Renaissance, Girolamo Fracastoro eut l'intuition que de minuscules particules vivantes, capables de se multiplier et invisibles à l'œil nu, étaient la cause de certaines maladies, et que ces particules pouvaient passer d'un individu à l'autre " par contact direct ou indirect, voire même sans contact, sur de longues distances ", mais il n'en avança strictement aucune preuve expérimentale [7]. Le jésuite allemand Athanase Kircher « crut » bien voir dans le sang des victimes de l'épidémie de peste de Rome et Naples de 1656 des « vermicules » qu'il considéra comme responsables de la maladie, mais son microscope ne dépassant pas un facteur de grossissement de 32, il ne vit probablement pas les coccobacilles responsables de la peste d'une taille comprise entre 1 et $5 \mu$ [14]. Cependant, l'idée était dans l'air. Près de 250 ans plus tard, Pierre Bulliard (1782), à la suite de plusieurs expériences remarquables, écrivait qu'une moisissure ne pouvait survenir que de la graine d'un individu de la même espèce [12], et Marcus von Plenciz (1792), reprenant les idées de ses prédécesseurs, formulait une théorie allant à l'encontre du paradigme toujours en vigueur à l'époque de la "génération spontanée » ou hétérogénie. Elle impliquait, à l'origine des maladies contagieuses, la présence d'« animalcules » invisibles à l'œil nu, vivant dans le sol ou dans l'eau, véhiculés et non plus générés par l'air, spécifiques de chaque maladie et d'un type $\mathrm{d}$ 'hôte et capables de se reproduire très rapidement en dehors du corps du patient dès qu'ils trouvaient un milieu favorable. Les notions de temps d'incubation, d'infection latente, d'infection mixte et même d'immunité et de chimiothérapie figurent dans son traité Opera-Medico-Physica paru en 1762 dans lequel Plenciz affirme également que les principes qu'il énonce sont communs à toutes les maladies infectieuses, qu'elles soient humaines, animales ou 
végétales [31]. Comme cela était à prévoir, médecins et savants refusèrent les idées de ce pionnier et continuèrent de considérer comme seuls agents pathogènes acceptables les vapeurs toxiques ou " miasmes » provenant des matières organiques en décomposition. Il est difficile de mesurer aujourd'hui l'importance de l'apport de Plenciz aux connaissances actuelles en matière de maladies infectieuses et de micro-organismes pathogènes, mais ses travaux et ses conclusions étaient indiscutablement en avance sur son temps d'un bon siècle, ce qui explique en partie le fait qu'ils n'aient rencontré que très peu d'échos. Il fallut attendre le début du XIX ${ }^{\mathrm{e}}$ siècle pour que les choses commencent à changer avec les prises de position de Théodore Schwann [34] et de Blondeau de Carolles [16], en faveur des idées de Plenciz, et que la théorie de la " génération spontanée » soit sérieusement remise en question avant de devenir peu à peu minoritaire dans la communauté scientifique.

C'est à partir de 1859-1860 que Pasteur commença ouvertement à la combattre et il finit par la mettre bas par ses célèbres expériences de 1863 que le film passe totalement sous silence [20]. Les scénaristes se contentent en effet d'illustrer la validation de la théorie des germes en montrant Pasteur recommandant, dans une sorte de pamphlet dont l'existence n'est pas avérée, une hygiène minutieuse des mains et la stérilisation des instruments médicaux, particulièrement ceux destinés à être employés en gynécoobstétrique. Cette recommandation est présentée dans le film comme étant à l'origine de l'assassinat d'un médecin qui a eu le tort de ne pas l'avoir suivie, par un homme qui juge le médecin responsable du décès de sa femme morte d'une fièvre puerpérale. L'affaire fait grand bruit et attire sur le savant les foudres de Napoléon III et celles de l'Académie de médecine.

Cette première et longue séquence sur la prophylaxie de la fièvre puerpérale, en début de film, a probablement été inventée de toutes pièces et inspirée par les vies de Holmes et Semmelweis. Alors que les microbes n'avaient toujours pas d'existence officielle, le médecin américain Wendel Holmes, dans les années 1840, avait en effet observé que les mains des médecins et des sages-femmes transmettaient « quelque chose » aux parturientes qui pouvait les tuer [13], tandis qu'un médecin hongrois, Ignác Fülöp Semmelweis, attirait l'attention sur la contagiosité de la fièvre puerpérale (1843) et recommandait fortement de se laver les mains à l'eau de chaux chlorée afin d'éviter les risques de contamination directe ou croisée entre accouchées par l'intermédiaire des soignants et des médecins, notamment lorsque ces derniers venaient de pratiquer une autopsie [35]. Les scénaristes mettent, en 1860, dans la bouche et les écrits de Pasteur, les mêmes recommandations que celles prônées par Holmes et Semmelweis sans tenir compte du fait que Pasteur, à cette date, ne s'intéressait pas particulièrement au lavage des mains et aux autres mesures d'asepsie qu'il ne préconisera véritablement qu'en 1878 , devant l'Académie de médecine où elles furent accueillies d'ailleurs avec scepticisme [27]. En établissant ainsi scientifiquement les bases de l'asepsie, Pasteur faisait écho à celles de l'antisepsie par l'eau phéniquée préconisée et mise en œuvre une dizaine d'années auparavant par Joseph Lister [17] qui eut le fair-play de reconnaître, dans une lettre envoyée au scientifique français en 1874, que ses réflexions sur l'antisepsie devaient beaucoup aux travaux de Pasteur sur la fermentation lactique. On peut certes s'étonner aujourd'hui de l'hostilité rencontrée dans l'ensemble du monde médical vis-à-vis d'une pratique aussi simple et sans risque que le lavage des mains et l'immersion dans l'eau bouillante des instruments médicaux et chirurgicaux, mais il ne faut pas oublier que cette hostilité et ce rejet procédaient d'une certaine logique à une époque où la théorie de la "génération spontanée ", bien que récemment invalidée par les expériences de Pasteur, gardait encore de nombreux adeptes.

Suite à sa victoire sur les tenants de l'hétérogénie, Pasteur n'a bien entendu jamais été réduit au silence et exilé à Arbois par Napoléon III pour les faits qui sont rapportés dans le film. Ce fils d'un sergent de la célèbre garde de Napoléon I entretenait bien au contraire d'excellents rapports avec le couple impérial [15] et se vit même confié par l'Empereur la tâche de résoudre les problèmes posés aux viticulteurs par les maladies du vin dues à des fermentations irrégulières et pathologiques. Le savant choisit naturellement les vignes de la région d'Arbois d'où il était originaire comme terrain d'étude et travailla sur ce sujet pendant deux ans au cours desquels, en s'inspirant des travaux d'Appert et d'autres, il mit au point une technique de stérilisation partielle du vin connue sous le nom de pasteurisation qui sera assez rapidement abandonnée dans cette indication, mais appliquée plus tard à d'autres boissons et notamment au lait par Franz von Soxlhet dès 1866 .

\section{Pasteur pionnier de la vaccination}

L'utilisation de la vaccine, beaucoup plus sûre que la variolisation pratiquée depuis très longtemps dans les pays ottomans et en Inde, existait, certes, depuis le XVIII ${ }^{\mathrm{e}}$ siècle et s'était répandue dans toute l'Europe, mais sans que ceux qui l'utilisaient ne comprennent le principe même de ce qu'ils faisaient et les mécanismes d'induction de la protection ainsi obtenue. Bien que considéré comme l'inventeur de la vaccination, Pasteur ne les comprenait pas davantage et s'imaginait que les souches atténuées qu'il utilisait pour fabriquer ses vaccins épuisaient certaines réserves de l'organisme du sujet vacciné, ce qui empêchait par la suite les bactéries virulentes responsables de la maladie de se développer [22]. Plus tard, lorsqu'il constata que cette explication était probablement erronée, il y renonça au profit d'une 
autre idée, tout aussi fausse que la précédente, bien qu'un peu plus proche de la réalité, faisant appel à la sécrétion par les bactéries à virulence atténuée servant à vacciner d'une substance empêchant par la suite les bactéries virulentes de se développer [21]. Atténuer les souches de bactéries pour produire des vaccins contre toutes les maladies infectieuses animales et humaines fut une des grandes idées de Pasteur et les différents modes d'atténuation de ces souches (chauffage, exposition à l'oxygène de l'air, passage sur animaux résistants ou peu sensibles) un des principaux sujets de recherche de la dernière partie de sa vie [28]. En raison de ses idées sur les mécanismes mis en jeu lors d'une vaccination et de son adhésion à la doctrine philosophique du vitalisme [32] qui impliquait une stricte séparation entre le vivant et la matière inanimée - jusqu'à ce que l'inutilité de cette barrière, ajoutait-il, ait été démontrée de manière expérimentale - Pasteur rejetait totalement l'idée que l'on puisse se servir de bactéries tuées pour fabriquer un vaccin, tout comme il n'avait pas jugé bon, lors de ses études sur les fermentations, de s'intéresser aux ferments solubles, enzymes et autres diastases pour ne se concentrer que sur le micro-organisme lui-même, en tant qu'être vivant.

Le premier vaccin fabriqué par l'homme le fut contre le choléra des poules en 1879 , à partir de souches atténuées par vieillissement de la bactérie responsable de cette enzootie [23]. Ces souches avaient été données à Pasteur par le vétérinaire Henri Toussaint qui avait été un des tout premiers à réussir à cultiver la bactérie. Suivirent, en 1881, un vaccin contre le charbon [26] qui était à l'époque un véritable fléau sanitaire et économique, puis contre le rouget du porc (1883) et enfin contre la rage en 1885 .

Le portrait de Napoléon III à peine décroché et remplacé par celui de Thiers, les scénaristes qui ont choisi de faire l'impasse sur les maladies du vin et des vers à soie et sur dix ans de la vie de Pasteur, nous présentent d'emblée, dans la seconde partie du film, une séquence peu crédible relatant la visite faite à Arbois par un certain Dr Radisse, personnage fictif incarnant un membre de l'Académie de médecine, venu constater, au nom de cette institution, l'absence de mortalité due au charbon chez les moutons vaccinés à la chaîne par Pasteur et pâturant dans la région. Radisse, qui dénie farouchement toute efficacité au vaccin de Pasteur, conclut son rapport en assurant que la région d'Arbois est « naturellement » vierge de la bactérie et invite tous les éleveurs de France à y venir avec leurs troupeaux. Le spectateur a bien du mal à suivre et à comprendre la manœuvre de Radisse pour discréditer Pasteur et l'enjeu de la séquence présentée par la suite, car le film ne donne aucune indication concernant les particularités de la transmission de la bactérie charbonneuse et le phénomène de sporulation qui la caractérise en lui permettant de rester très longtemps présente dans le sol pour resurgir périodiquement, donnant lieu à la croyance que certaines régions et certains champs étaient maudits. La paternité de la découverte et de l'interprétation de ce phénomène fut l'objet d'une très vive polémique entre Pasteur, qui en revendiquait la priorité, et Robert Koch dont le nom n'est même pas mentionné dans le film, bien qu'il soit considéré en Allemagne comme le fondateur de la bactériologie, alors que le nom de Pasteur y est essentiellement associé à la vaccination contre la rage. La rivalité entre Koch et Pasteur sur ce point et quelques autres fut pourtant extrêmement vive, entretenue par le nationalisme exacerbé de Pasteur après la défaite de Sedan et la perte de l'Alsace et de la Lorraine [30].

En réalité, c'est à la suite d'une déclaration de Pasteur devant l'Académie des sciences annonçant la mise au point par son équipe d'un vaccin efficace contre le charbon à partir de souches dont la virulence avait été atténuée par la chaleur et l'oxygène de l'air, qu'un vétérinaire de la région de Melun, le Dr Rossignol, tout à fait sceptique quant à l'efficacité de ce vaccin, proposa à Pasteur, au nom de la Société des agriculteurs de France, d'en faire une démonstration publique dans sa ferme de Pouilly-le-Fort en vaccinant un lot de 25 moutons avant de leur injecter, ainsi qu'à un lot équivalent d'animaux non vaccinés, le sang d'une brebis morte du charbon [24]. C'était à peu de choses près le protocole qu'avait suivi un an auparavant le vétérinaire Henri Toussaint pour tester un vaccin contre le charbon fondé sur l'utilisation de bactéries traitées par la chaleur, puis par le phénol, dont il avait été sommé de dévoiler le mode de fabrication lors d'une séance de l'Académie des sciences [36,37]. Pasteur, en vacances, n'assistait pas à cette séance, mais le vétérinaire Henri Bouley, qui en avait été rapporteur, lui avait communiqué la teneur de la note de Toussaint, sachant que Pasteur travaillait sur le sujet.

L'expérience de Pouilly est présentée à l'écran dans une atmosphère de kermesse, ce qui fut un peu le cas, car nombre de journalistes, de personnalités de tous ordres et de curieux firent le déplacement, mais certainement pas Joseph Lister que l'on voit pourtant congratuler Pasteur, lequel congratule à son tour Lister en une longue embrassade..., qui n'eut lieu, dans la réalité, que dix bonnes années plus tard, à la Sorbonne, au cours du jubilé de Pasteur, en présence du président de la République Sadi Carnot [33].

En dehors de cette entorse faite à l'Histoire, c'est surtout la seconde embrassade que donne ensuite Pasteur à son assistant Émile Roux qui retient l'attention. Un spectateur averti peut être tenté d'y voir, de la part d'un homme d'ordinaire si réservé et respectueux des hiérarchies, plutôt que la joie d'un complice félicitant un autre complice pour avoir réussi un joli coup, une allusion au fameux secret de Pouilly. Tout en laissant croire qu'il n'avait utilisé que des souches atténuées par chauffage et exposition à l'oxygène de l'air, Pasteur, qui n'était pas tout à fait sûr de la stabilité et de l'efficacité de son vaccin, avait utilisé sans le dire un vaccin préparé en secret par Roux et Chamberland sur le modèle de celui de Toussaint, en remplaçant le phénol par du 
bichromate de potassium. De par la volonté même de Pasteur, ce secret ne devait pas être levé avant que le vaccin atténué, conçu au départ par Pasteur, ne soit totalement au point. Il fut levé malgré tout dès 1883 par Roux en raison de contraintes commerciales liées à la fabrication industrielle $\mathrm{du}$ vaccin, mais de façon si discrète que le doute persista pendant de longues années avant que ce manquement manifeste à la déontologie d'un chercheur qui se voulait exemplaire ne soit plus l'objet de controverses. Savoir si cette manœuvre à la limite de l'honnêteté avait pour unique but d'empêcher Henry Toussaint de revendiquer la priorité quant à la mise au point et à l'utilisation d'un vaccin contre le charbon, comme le pense A. Cadeddu, est une question qui reste posée $[1,18]$.

Dans un tout autre registre, l'astuce de mise en scène qui consiste à faire croire dans un premier temps que les moutons vaccinés sont malades ou morts, alors qu'ils ne sont qu'endormis et se réveillent brusquement à l'aboiement d'un chien, fait son effet sur les spectateurs qui ne connaissent pas la fin de l'histoire. Mais le protocole de l'expérience de Pouilly et la chronologie de ses différentes étapes sont si mal précisés dans le film qu'il est à craindre que les spectateurs s'imaginent que les moutons aient été vaccinés la veille et aient reçu pratiquement en même temps le sang contenant la bactérie virulente destinée à les tester. En réalité, l'expérience de Pouilly se déroula sur un bon mois, et les moutons vaccinés par deux injections à 12 jours d'intervalle l'une de l'autre furent testés 12 jours après la dernière injection [26].

\section{Vaccination contre la rage}

La dernière partie du film est consacrée à la vaccination contre la rage. La scène au cours de laquelle Charbonnet s'injecte le virus $^{1}$ est fictive, comme l'est le personnage. Celle qui montre Louis Pasteur vaccinant le jeune Joseph Meister sans être autorisé à le faire, car il n'était pas médecin, est tout aussi fictive que la précédente, et il est bien clair, maintenant, que la vaccination n'a jamais été effectuée par Pasteur lui-même, mais par le Dr Grancher, pédiatre de l'hôpital Necker, qui lui était tout dévoué. Roux aurait pu se charger des injections, mais il s'y était refusé considérant que le cas du jeune garçon était douteux et la balance bénéfice/risque - si l'on peut employer cette expression anachronique - trop incertaine.

\footnotetext{
${ }^{1}$ La notion de virus filtrant ou encore d'infrabactéries comme les appelait Pasteur, c'est-à-dire d'agents pathogènes capables de traverser le filtre de porcelaine de Chamberland, ne fut vraiment acquise qu'en 1892. Le terme virus a longtemps été employé pour désigner n'importe quel organisme pathogène, que ce soit une bactérie, un parasite ou un virus filtrant. L'appartenance des virus « sensu stricto » au monde du vivant est toujours discutée..., ce qui n'aurait pas manqué d'intéresser au plus haut point l'adepte du vitalisme qu'était Pasteur.
}

Contrairement à ce qui est montré dans le film, le jeune Meister n'était probablement pas atteint d'hydrophobie lorsqu'il reçut les premières injections. La question de savoir si le chien qui l'avait mordu était enragé reste posée. Pasteur se la posa lui-même puisqu'il termina la vaccination en faisant procéder à l'injection d'une culture particulièrement virulente du virus pour lever le doute et avoir la certitude que son vaccin était efficace [2]. Le jeune garçon avait été mordu à 14 reprises par un chien considéré comme enragé, mais sans autre preuve que celle d'avoir trouvé de la paille et du bois dans l'estomac de l'animal, ce que l'on considérait encore, mais déjà de moins en moins à l'époque, comme significatif. Quoi qu'il en soit, chacun sait que le jeune Meister ne développa jamais la rage et devint, tout au moins pour l'histoire officielle, le premier être humain vacciné contre cette maladie jusque-là toujours mortelle. Toutefois, en consultant les carnets de laboratoire de Pasteur, tenus longtemps secrets par la volonté même du savant et consultables depuis peu avec des restrictions, on s'aperçoit que Meister n'était en fait que le troisième vacciné, le premier ayant survécu, car n'ayant probablement pas la rage, et la deuxième étant morte de la rage après une vaccination trop tardive [4].

Passés les premiers succès, l'efficacité et l'innocuité du vaccin antirabique furent rapidement remises en question après l'apparition des premiers échecs, tant par les journaux que par le monde médical et l'opinion. Pour se prouver à luimême qu'il avait raison contre tous et que sa méthode de vaccination était au point et sans réel danger, Pasteur demanda alors à Grancher de le vacciner puis de se faire vacciner par Adrien Loir qui était médecin et neveu de Pasteur par alliance, car Grancher venait de se piquer accidentellement avec l'aiguille d'une seringue contenant un broyat de moelle virulente. Grancher accepta sans hésitation de se faire vacciner, mais refusa de vacciner Pasteur. Loir, sollicité, refusa à son tour de vacciner son oncle, mais proposa de se faire vacciner lui-même à sa place et de servir ainsi en quelque sorte de cobaye. Viala le garçon de laboratoire, qui faisait presque partie de la famille et qui avait assisté à la scène, l'imita [4]. Les injections, faites secrètement, se répétèrent au fil des jours, Grancher vaccinant Loir, Loir vaccinant Grancher et l'un ou l'autre des deux médecins vaccinant Viala qui était chargé de préparer les seringues. Lorsqu'il se rendit compte de ce qui était en train de se passer, Émile Roux qui avait toujours considéré la technique de vaccination adoptée par Pasteur comme manquant de fiabilité, et pour qui, déontologie et éthique n'étaient pas de vains mots, entra dans une colère noire. En voulant démontrer sur lui-même l'innocuité de son vaccin, Pasteur n'avait fait cependant que rejoindre la cohorte des jeunes chercheurs débordant d'enthousiasme et des savants confirmés d'hier et d'aujourd'hui qui, pêchant par excès de confiance, n'hésitent pas à expérimenter sur eux-mêmes comme ce fut le cas du célèbre Schaudinn qui mourut des complications d'une 
amibiase intestinale contractée volontairement pour en observer l'évolution ou celui de l'étudiant en médecine Daniel Carrión qui décéda de la fièvre d'Oroya, forme septicémique hémolytique d'une bartonellose, après s'être inoculé un broyat de verruga pour prouver l'unicité de la maladie qui porte son nom.

Si le mérite d'avoir réussi à fabriquer un vaccin antirabique efficace utilisable chez l'homme revient bien à Pasteur, on ne peut plus passer sous silence aujourd'hui le rôle joué par Galtier dans la fabrication et dans le mode d'utilisation de ce vaccin. Après que Duboué eût déduit de ses observations que le virus cheminait très lentement le long des voies nerveuses à partir de la morsure pour gagner le système nerveux central et provoquer la mort [6], c'est en effet Victor Galtier qui eut l'idée de mettre à profit ce délai de deux à huit semaines en moyenne avant l'issue fatale pour essayer d'agir sur le virus et le détruire, sans toutefois préciser et proposer le moyen d'y parvenir [8]. Ce vétérinaire ardéchois, Lyonnais d'adoption, découvrit également la possibilité de se servir du lapin comme d'un animal de laboratoire, « commode et inoffensif », pour étudier la rage et la diagnostiquer à partir de prélèvements [9]. Pasteur utilisera d'innombrables moelles desséchées de lapin pour fabriquer son fameux vaccin [25]. Galtier, de son côté, suite à des échecs répétés de transmission du virus rabique par des broyats de tissus nerveux de chien enragé, commit l'erreur de croire que ce virus ne se trouvait, chez le chien, qu'au niveau de sa gueule et dans ses glandes salivaires et sublinguales. Tout en utilisant très largement ses idées, Pasteur traita avec un certain mépris l'ensemble des travaux du vétérinaire et utilisa cette erreur pour disqualifier à tort la prétention de Galtier d'avoir réussi à immuniser un mouton en lui injectant de la salive de chien enragé, réalisant ainsi, en fait, la première vaccination antirabique préventive de l'Histoire [10,11] (1881-1882). Nocard et Roux confirmèrent pourtant dès 1884 et également en 1888 la validité des résultats de Galtier et la possibilité de vacciner moutons et chèvres par voie intraveineuse avec de la salive de chien enragé [19].

\section{Louis Pasteur : l'homme et son image dans le public}

Peu de gens savent que Pasteur souffrait d'une hémiplégie gauche, séquelle d'un accident vasculaire cérébral survenu à l'âge de 46 ans, alors qu'il travaillait sur les maladies des vers à soie. Dieterle le filme somnolent lors d'une des dernières séquences après pratiquement quatre nuits de veille. Ces images sont manifestement faites pour émouvoir le spectateur en montrant la fatigue, voir l'épuisement de ce grand travailleur dont la devise était « Laboremus » et dont les engagements et la combativité n'eurent d'égale que l'importance des résultats de ses travaux. Elles nous font également oublier son carac- tère difficile, autoritaire, volontiers agressif, « arrogant, inflexible, voire dogmatique » [4] et presque antipathique qui lui coûta son poste d'administrateur de l'École normale supérieure ainsi que sa sensibilité aux honneurs et à la reconnaissance des grands de ce monde [30].

«Il n'existe pas une catégorie de sciences auxquelles on puisse donner le nom de sciences appliquées. Il y a la science et les applications de la science, liées entre elles comme le fruit à l'arbre qui l'a porté », aimait rappeler le savant, comme s'il était conscient d'être l'exemple même de ce qu'un travail inlassable, cohérent et centré sur un ensemble de tâches bien précises, est un facteur de réussite, au même titre et peut-être même davantage que l'inventivité ou le génie. Plus qu'un découvreur, Pasteur fut, en effet, une sorte de chef d'orchestre de la microbiologie naissante, sachant capter, utiliser, faire la synthèse de tous les talents, utiliser et marier entre elles toutes les idées et les découvertes qui se faisaient ou s'étaient faites dans ce domaine pour en tirer une musique harmonieuse et cohérente, même si certains lui reprochent de l'avoir fait à son seul profit, attirant sur lui toute la lumière, comme le font habituellement les chefs d'orchestre de renom, et laissant aux autres, qu'ils soient pionniers, contemporains ou collaborateurs, la grisaille de l'anonymat ou l'ombre de l'oubli. Même s'il y a une bonne part de vérité en cela, Louis Pasteur, au-delà des polémiques, reste, pour beaucoup, l'accoucheur d'une nouvelle science qui, en moins d'un siècle, a révolutionné l'espérance de vie d'une très grande partie de l'Humanité.

Un grand nombre de scientifiques dans le monde ont eu et ont toujours de l'admiration et beaucoup de respect pour ce Français considéré comme un maître de l'expérimentation. Carlos Chagas, qui n'hésitait pas à faire appel au dithyrambe, écrivait de ses travaux : "La grande réussite de Pasteur... marqua le début d'une nouvelle ère de triomphes et l'aube d'un jour splendide dans l'histoire de la science d'Hippocrate » [3]. Pour le grand public, qui connaît bien son nom et mal son œuvre, Pasteur est devenu une icône, l'archétype du savant modèle, altruiste, désintéressé et sans défaut qui a vaincu la rage et que sa famille, ses collaborateurs et les promoteurs de la $\mathrm{III}^{\mathrm{e}}$ République ont largement contribué à créer, car ils en avaient besoin ; mais, finalement, peu de gens mettent à la première ligne de son crédit le fait qu'il a scientifiquement invalidé le paradigme de la "génération spontanée » et permis ainsi la naissance d'une nouvelle science.

Par-delà les luttes qu'il dut mener et l'hostilité du monde médical qu'il dut affronter pendant une bonne partie de sa vie, Pasteur eut, malgré tout, la chance de voir, de son vivant, triompher toutes ses idées et d'en recevoir son comptant d'honneurs et de gloire. Membre de quatre académies (sciences, médecine, vétérinaire et Académie française), lauréat d'innombrables prix, directeur d'un Institut qu'il finit par baptiser lui-même de son nom [33] et qui le 
pérennise, il repose aujourd'hui, après des funérailles nationales, au cœur de Paris, dans une tombe digne des plus grands pharaons où une sorte de culte lui est rendu chaque année le jour anniversaire de sa mort par les représentants de ce qui est devenu et qu'il est convenu d'appeler la « grande famille pasteurienne » [29].

\section{Conclusions}

Les recherches incessantes et inlassables de Pasteur visant à rendre intelligibles des phénomènes qui paraissaient jusquelà inexpliqués et qui posaient depuis des siècles des problèmes insolubles tant aux médecins qu'aux patients est un exemple, pour tous ceux qui pourraient en douter, que seul un travail constant et engagé au service d'une idée peut mener à un résultat gratifiant.

« Jeunes hommes - médecins et scientifiques du futurne vous laissez pas impressionner par un scepticisme apparent, ni décourager par la tristesse de certaines heures qui se glissent entre les nations. Ne vous fâchez pas contre vos adversaires, car aucune théorie scientifique n'a jamais été acceptée sans opposition. Vivez dans la paix sereine des bibliothèques et des laboratoires ».

Tel est le message, que les scénaristes et le réalisateur ont voulu faire passer en choisissant, pour terminer leur film, ces deux phrases prononcées par un Pasteur volontiers sentencieux et donneur de leçons, adressé aux jeunes gens qui se pressaient au balcon du grand amphithéâtre de la Sorbonne lors de son jubilé. Ces deux phrases, dans la réalité, étaient agencées de manière un peu différente et faisaient partie d'un discours écrit certes par Pasteur, mais lu par son fils [4]. Il n'est pas certain que Pasteur lui-même ait toujours suivi ce conseil et que cette phrase caractérise au mieux ce que fut sa vie.

Remerciements : CTDR est Bolsista de Produtividade em Pesquisa du Conseil national pour le développement scientifique et technologique (CNPq, Brésil) et Cientista do Nosso Estado de la fondation Carlos-Chagas-Filho pour le soutien à la recherche dans l'État de Rio de Janeiro (FAPERJ). Le Laboratório de Pesquisa em Malária est un laboratoire associé de l'INCT (Instituto Nacional de Ciência e Tecnologia) au Brésil

Liens d'intérêts : Les auteurs déclarent ne pas avoir de liens d'intérêts.

\section{Références}

1. Cadeddu A (1987) Pasteur et la vaccination contre le charbon. Une analyse historique et critique. Hist Philos Life Sci 9:255-76
2. Cadeddu A (1996) Aux origines de la vaccination pasteurienne : la rage. In: Moulin AM (ed) L'aventure de la vaccination. Fayard, Paris, pp 168-84

3. Chagas C (1903) Estudos hematológicos no impaludismo in Tese: Valor dos estudos de hematologia patológica. Instituto de Manguinhos, Rio de Janeiro

4. Debré P (1994) In Louis Pasteur. Flammarion Ed., Paris, 562 p

5. Dieterle W (1936) La vie de Louis Pasteur (The Story of Louis Pasteur), First National Productions, 87 minutes. https://www. youtube.com/watch? $\mathrm{v}=\mathrm{gcMBpKp} 8 \mathrm{jcQ}$

6. Duboué PH (1879) De la physiologie pathologique et du traitement rationnel de la rage. Suite d'études de pathogénie. V. Adrien Delahaye Ed., Paris, $147 \mathrm{p}$

7. Fracastoro G (1546) De contagione et contagiosis morbis venise. In: Fracastoro G, Wright WC (eds) Hieronymii Fracastorii de contagione, Libri III, translation and notes. 1930. G.P. Putnam's Sons, New York, London

8. Lépine P (1969) Galtier et les recherches sur la rage. Bull Acad Med. 153:78-81

9. Galtier V (1879) Étude sur la rage. Rage du lapin. CR Acad Sci 89:444-46

10. Galtier V (1881) Transmission du virus rabique. Bull Acad Med 1881:90-4

11. Galtier V (1904) Une page d'histoire. Découverte de l'immunité rabique. Vaccination antirabique par injection intraveineuse. J Med Vet Zoot (Lyon) 55:274-77

12. Gilbert E (1952) Jean-François Bulliard as precursor of Pasteur. Mycopath Mycol Appl 6:237-9

13. Holmes OW (1885) Puerperal fever as a private pestilence. Tickmar and Fields Ed., Boston, $60 \mathrm{p}$

14. Kircher A (1659) Scrutinium physico-medicum contagiosae Luis seu pestis. Schüreri \& Gotzii Ed., 532 p

15. Lemaire JF (1996) Napoléon III et Pasteur. Revue du Souvenir Napoléonien 407:19-27

16. Lemay P (1952) Blondeau de Carolles, an immediate and unknown precursor of Pasteur. Prog Med (Paris) 80:189

17. Lister $J$ (1867) On the antiseptic principle in the practice of surgery. Br Med J 2:246-8

18. Loir A (1936) Le mouvement sanitaire. 141: 398 (consultable sur Gallica)

19. Nocard E, Roux E (1888) Expériences sur la vaccination des ruminants contre la rage par injections intraveineuses du virus rabique. An Instit Pasteur 2:341-53

20. Pasteur L (1861) Mémoire sur les corpuscules organisés qui existent dans l'atmosphère. Examen de la doctrine des générations spontanées. Euvres complètes T 2, p 223

21. Pasteur L (1880) Sur le choléra des poules ; études de la condition de la non-récidive de la maladie et de quelques autres de ses caractères. CR Acad Sci 90:957

22. Pasteur L (1880) Sur les maladies virulentes, et en particulier sur la maladie appelée vulgairement choléra des poules. Euvres complètes T 6,p 301

23. Pasteur L (1880) De l'atténuation du virus du choléra des poules. CR Acad Sci 91:673-80

24. Pasteur L (1881) Compte rendu sommaire de L. Pasteur des expériences faites à Pouilly-le-Fort, près Melun, sur la vaccination charbonneuse en commun avec MM. Chamberland et Roux. CR Acad Sci 92:1378-83

25. Pasteur L (1885) Méthode pour prévenir la rage après morsure L. Pasteur. Cr Acad Sci 101:765-774.

26. Pasteur L, Chamberland C, Roux E (1881) De la possibilité de rendre les moutons réfractaires au charbon par la méthode des inoculations préventives. CR Acad Sci 92:662-5

27. Pasteur L, Joubert J, Chamberlain C (1878) La théorie des germes et ses applications à la médecine et à la chirurgie. G. Masson Ed., $23 \mathrm{p}$ 
28. Pasteur L, Chamberland C, Thuillier L, Roux E (1882) De l'atténuation des virus. IV ${ }^{e}$ Congrès international d'hygiène et de démographie. Genève 1:127-49

29. Perrey C (2005) Les figures du sacré à l'Institut Pasteur. L'Homme 175-176:345-68

30. Perrot A, Schwartz M (2014) Pasteur et Koch, un duel de géants dans le monde des microbes. Odile Jacob Sciences Ed., Paris, 227 p

31. Plenciz MA (1762) Opera medico-physica. De contagio. Volume 1. Trattner Ed., Vienne, $276 \mathrm{p}$

32. Pinet P (2005) Pasteur et la philosophie (SIII : le vitalisme de Pasteur). L'Harmattan Ed., Paris, 234 p

33. Salomon-Bayet C (1998) La gloire de Pasteur. Romantisme 100:159-69
34. Schwann T (1837) Vorläufige Mitteilung betreffend Versuche über Weingährung und Fäulniss. Annalen der Physik und Chemie XLI:184-93

35. Semmelweis IF (1861) Die Aetiologie, der Begriff und die Prophylaxis des Kindbettfiebers. CA Hartlem's Verlag Ed. Pest, Wien, Leipzig, $543 \mathrm{p}$

36. Toussaint H (1880) Note contenue dans un pli cacheté et relative à un procédé pour la vaccination du mouton et du jeune chien (Note de H. Toussaint présentée par M. Bouley). CR Acad Sci 91:303

37. Toussaint H (1880) De l'immunité pour le charbon acquise à la suite d'inoculations préventives. CR Acad Sci T 91:135-7 